\title{
Abnormal cross-frequency coupling in the tinnitus network
}

\author{
Ilya Adamchic ${ }^{1 *}{ }^{*}$, Berthold Langguth ${ }^{2,3}$, Christian Hauptmann ${ }^{1}$ and Peter A. Tass ${ }^{1,4,5}$ \\ 1 Jülich Research Center, Institute of Neuroscience and Medicine, INM-7, Neuromodulation, Jülich, Germany \\ ${ }^{2}$ Department of Psychiatry and Psychotherapy, University of Regensburg, Regensburg, Germany \\ ${ }^{3}$ Interdisciplinary Tinnitus Center, University of Regensburg, Regensburg, Germany \\ ${ }^{4}$ Department of Neurosurgery, Stanford University, Stanford, CA, USA \\ ${ }^{5}$ Department of Neuromodulation, University of Cologne, Cologne, Germany
}

\section{Edited by:}

Edward W. Large, Florida Atlantic

University, USA

Reviewed by:

Sven Vanneste, University of Texas at Dallas, USA

Dirk De Ridder, University of Otago, New Zealand

\section{*Correspondence:}

Ilya Adamchic, Jülich Research

Center, Institute of Neuroscience and Medicine, INM-7,

Neuromodulation, Leo-Brandt-Str., 52425 Jülich, Germany

e-mail: i.adamchic@gmail.com
Neuroimaging studies have identified networks of brain areas and oscillations associated with tinnitus perception. However, how these regions relate to perceptual characteristics of tinnitus, and how oscillations in various frequency bands are associated with communications within the tinnitus network is still incompletely understood. Recent evidence suggests that apart from changes of the tinnitus severity the changes of tinnitus dominant pitch also have modulating effect on the underlying neuronal activity in a number of brain areas within the tinnitus network. Therefore, in a re-analysis of an existing dataset, we sought to determine how the oscillations in the tinnitus network in the various frequency bands interact. We also investigate how changes of tinnitus loudness, annoyance and pitch affect cross-frequency interaction both within and between nodes of the tinnitus network. Results of this study provide, to our knowledge, the first evidence that in tinnitus patients, aside from the previously described changes of oscillatory activity, there are also changes of cross-frequency coupling (CFC); phase-amplitude CFC was increased in tinnitus patients within the auditory cortex and the dorsolateral prefrontal regions between the phase of delta-theta and the amplitude of gamma oscillations (Modulation Index [MI] 0.17 in tinnitus patients vs. 0.08 in tinnitus free controls). Moreover, theta phase in the anterior cingulate region modulated gamma in the auditory (MI 0.1) and dorsolateral prefrontal regions (MI 0.19). Reduction of tinnitus severity after acoustic coordinated reset therapy led to a partial normalization of abnormal CFC. Also treatment induced changes in tinnitus pitch significantly modulated changes in CFC. Thus, tinnitus perception is associated with a more pronounced CFC within and between nodes of the tinnitus network. CFC can coordinate tinnitus-relevant activity in the tinnitus network providing a mechanism for effective communication between nodes of this network.

Keywords: tinnitus pitch, oscillations, delta band activity, alpha rhythm, gamma band activity, coordinated reset neuromodulation, cross frequency coupling

\section{INTRODUCTION}

Phantom auditory perception refers to the conscious awareness of an internally generated sensory percept when no matching auditory stimulus is present (Eggermont, 2003; Snow, 2004; Møller et al., 2010). Synchronization between different cortical areas in the tinnitus network plays an important role in the pathophysiological basis of tinnitus (Schlee et al., 2008, 2009). Synchronization at single frequencies between distant tinnitus hubs is thought to be relevant to how these regions interact within the tinnitus network, and such interactions have been well studied (Schlee et al., 2008, 2009; Vanneste et al., 2011; Silchenko et al., 2013). However, it remains unclear how different frequency bands interact both within and between various nodes of the tinnitus network. Compared to the synchronization at single frequencies cross-frequency coupling (CFC) would allow for much more complex corticocortical interactions and has also been extensively studied in various brain signals and across distant cortical regions (Shils et al., 1996; Schack et al., 2002; Buzsaki and Draguhn, 2004;
Palva et al., 2005; Canolty et al., 2006; Palva and Palva, 2007; Darvas et al., 2009). CFC in the brain suggests that non-linear interactions can play a crucial role in the coordination of complex cortical computation. Here, we investigate cross-frequency phase-amplitude coupling both within and between hubs of the tinnitus network, using a comodulation analysis introduced by Tort et al. (2008). Previous encephalographic (EEG), magnetoencephalographic (MEG) and electrocorticographic studies have demonstrated the possibility of high frequencies (e.g., in the gamma band) being modulated by lower frequencies (Mormann et al., 2005; Canolty et al., 2006; Osipova et al., 2008). For example, it has been shown that high-frequency gamma power $(30-70 \mathrm{~Hz})$ is phase-locked to alpha oscillations $(8-13 \mathrm{~Hz})$ in the ongoing MEG signals (Osipova et al., 2008). Another study showed that theta phase modulates gamma $(30-50 \mathrm{~Hz})$ amplitude (Lakatos et al., 2005). Therefore, we focused our analysis on cross-frequency phase-amplitude interactions between frequencies ranging from 1 to $30 \mathrm{~Hz}$, covering the delta, theta, alpha 
and beta bands, with frequencies from 30 to $48 \mathrm{~Hz}$, i.e., gamma band.

The experience of tinnitus involves sensory and emotional components that are partially interdependent (Jastreboff, 1990; Weisz et al., 2005; De Ridder et al., 2011a). Recently there has been a growing interest in how the separate psychoacoustic characteristics of the tinnitus signal (e.g., loudness, pitch, noise vs. pure tone) are encoded and how their changes affect neuronal processing in the tinnitus aversive network (van der Loo et al., 2009; Vanneste et al., 2010; Adamchic et al., 2012a). Traditionally therapeutic interventions have been evaluated either in their effects on tinnitus distress or on tinnitus loudness (Aazh et al., 2008; De Ridder and Vanneste, 2011; Landgrebe et al., 2012; Tass et al., 2012). However, recent results suggest existence of an interdependence between perceptual characteristics of tinnitus and the related handicap. In addition to reduction of subjective tinnitus loudness that has been shown to have a strongly influence on tinnitus distress, changes in other characteristics like tinnitus pitch may have an important modulating effect as well (Bartels et al., 2007; Adamchic et al., 2012a; Tass et al., 2012). In a recent article, we reported that reduction of both subjectively perceived tinnitus loudness and tinnitus annoyance after acoustic CR neuromodulation (Tass, 2003; Tass and Popovych, 2012; Tass et al., 2012) significantly correlated with the modulus of the tinnitus pitch ratio (Adamchic et al., 2012a). In order to identify the neuronal correlates of the pitch change we divided patients into two groups: (i) a group with pronounced pitch change (PC), (ii) a group with no or minimal tinnitus pitch change (NPC), while keeping the relief of subjectively perceived tinnitus loudness and annoyance similar in both groups. Patients in the PC group had modulus pitch change ratio, that is, the absolute value of the pitch change ratio, of $<0.3$ and a reduction of VAS loudness (VAS-L)/VAS annoyance (VAS-A) of -26.9[17.5]/-25.7[15.2] [mean, (standard deviation)]. Patients in the NPC group had a modulus pitch change ratio of $>0.3$ and reduction of VAS-L/VAS-A of $-27.1[10.3] /-26.2[12.1]$. In patients with pronounced tinnitus pitch change there were significantly stronger treatment related changes of brain oscillatory activity in a distributed network of brain areas which included the left superior temporal cortex (AC), the dorsolateral prefrontal region (DLPFC), the left premotor cortex, and the anterior cingulate cortex (ACC). Changes of brain activity in the region of the superior temporal gyrus (Brodmann area 22; BA) in the gamma band correlated with the tinnitus pitch change ratio. These findings suggest that changes of different perceptual characteristics of tinnitus (pitch and loudness) may have modulating effect on each other, on tinnitus annoyance and on the underlying neuronal activity (Adamchic et al., 2012a) and fits with the hypothesis that tinnitus results from pathological oscillatory activity in various dynamic overlapping brain networks (Schlee et al., 2009; De Ridder et al., 2011a, 2014; Vanneste et al., 2011; Langguth et al., 2013; Silchenko et al., 2013). This finding also showed that a synchronous tinnitus related linkage is possible between DLPFC, AC and ACC, but the functioning of such linkage was not readily apparent. Therefore, in this study we investigated crossfrequency phase-amplitude coupling both within and between cortical areas that were previously shown to be part of the tinnitus network. Firstly we compared CFC in tinnitus patients and healthy controls and secondly we investigated the effects of treatment induced changes of the tinnitus loudness, annoyance and pitch.

We used high density EEG data recorded from 32 patients with chronic tonal tinnitus and tested for the existence of phaseamplitude CFC within as well as between three structures: (1) auditory cortex region, (2) dorsolateral-prefrontal region, and (3) anterior cingulate cortex. We hypothesized that tinnitus patients as compared to healthy controls show evidence of abnormal CFC across a broad range of frequencies, and that this CFC is changed by $\mathrm{CR}$ neuromodulation induced reduction of the tinnitus symptoms.

\section{METHODS}

\section{SUBJECTS}

In this study we re-analyzed EEG data recorded in tinnitus patients who participated in a multicentric randomized, controlled clinical trial on acoustic $\mathrm{CR}$ neuromodulation in the treatment of chronic subjective tonal tinnitus ["RESET study," ClinicalTrials.gov Identifier: NCT00927121 (Tass et al., 2012)]. Inclusion criteria, selection of patients and definition of the PC and NPC groups are described in detail in Adamchic et al. (2012a) and Tass et al. (2012). The baseline VAS-L/VAS-A in all 32 patients from both PC and NPC groups was 68.4[15.3]/66.3[17.5], their average age was 49.6[11.9] years and the pure-tone averages (the average hearing threshold level) of $1-4$ and $6-12 \mathrm{kHz}$ were 17.6[9.4] and 39.1[17.5], respectively (Adamchic et al., 2012a). The control group consisted of 16 healthy tinnitus-free subjects (10 men and 6 women). Their average age was 45.0[12.5] years and the pure-tone averages of $1-4$ and $6-12 \mathrm{kHz}$ were 12.73 [13.69] and 24.29[19.05], respectively.

\section{CR TREATMENT}

In the Tass et al. (2012) study patients were stimulated with acoustic CR neuromodulation for 12 weeks using a portable acoustic device and comfortable earphones. Each patient underwent two EEG recording sessions, the first one on the first treatment day before start of the treatment and the second at the 12-weeks follow up. EEG was recorded at a minimum 2-h break from stimulation. Subjectively perceived tinnitus loudness and tinnitus annoyance were assessed off-stimulation using a VAS-L and VASA (Adamchic et al., 2012b). The CR neuromodulation resulted in a significant and clinically relevant decrease of tinnitus severity as measured by VAS-L/VAS-A and TQ scores (Adamchic et al., 2012b,c; Tass et al., 2012).

\section{EEG DATA ACQUISITION AND DATA ANALYSIS}

EEG recordings were obtained in a dimly lit room in a Faraday cage from 128 surface electrodes (HydroCel Geodesic Sensor Net) referenced to $\mathrm{Cz}$. The EEG signals were amplified with a Net Amps 200 amplifier (Electrical Geodesis Inc, Eugene, USA), digitized at $1 \mathrm{kHz}$ and band-pass filtered from 0.1 to $400 \mathrm{~Hz}$. Recordings were made in an awake state with the subjects in alternating $2 \mathrm{~min}$ intervals with eyes closed and with eyes open and the eyes closed data was selected for further analysis. Each EEG recording was corrected for eye blink and movement artifacts using the surrogate model approach in BESA (Brain Electrical Source Analysis, MEGIS Software, 5.2 version) (Ille et al., 2002). Surface EEG was 
transformed into brain source activity using the source montage approach in BESA (Scherg et al., 2002). A source model was generated with regional neural sources placed in the regions of interest (ROI). Based on the results of Adamchic et al. (2012a), the source montage consisted of ROIs with sources placed in the regions of the left auditory cortex (AC), right dorsolateralprefrontal cortex (DLPFC) and anterior cingulate cortex (ACC). Talairach coordinates of these ROIs were: AC ( $\mathrm{x}-58, \mathrm{y}-45, \mathrm{z}$ 16), DLPFC (x 53, y 14, z 32), ACC (x 6, y 37, z 11). Additional probe-sources were placed into the occipital lobe, orbito-frontal region, into the area of the central sulcus in both hemispheres as well as into the left DLPFC and right AC. These sources outside the ROIs acted as a spatial filter and reduced the contribution of these regions to the ROI.

EEG signals were adaptively filtered using the data-driven empirical mode decomposition (EMD) approach where the basis functions are derived directly from the time-series itself (Huang et al., 1998). EMD provides a set of intrinsic mode functions (IMF) for signals from each source with each IMF corresponding to one of the co-existing time scales. EMD allows avoidance of drawbacks caused by the band pass filtering (Florin et al., 2010). Phases were extracted by Hilbert transformation for every IMF from 1 to $30 \mathrm{~Hz}$. The amplitude for high frequencies between 30 and $48 \mathrm{~Hz}$ was calculated using Hilbert transformation for each $1 \mathrm{~Hz}$ frequency step ( $4 \mathrm{~Hz}$ wide band pass filter). Frequency bands were defined as follows: delta $(1-4 \mathrm{~Hz})$, theta $(4-8 \mathrm{~Hz})$, alpha $(8-13 \mathrm{~Hz})$, gamma $(30-48 \mathrm{~Hz})$.

\section{ESTIMATION OF PHASE-AMPLITUDE COUPLING}

Given a set of IMFs for each of the source signals, we used the modulation index (MI) and comodulation analysis introduced by Tort et al. (2008) to calculate hierarchical phase-amplitude coupling (Lakatos et al., 2005; Tort et al., 2008, 2010). The MI represents a normalized measure of CFC between 2 frequency ranges of interest: a phase-modulating lower frequency $(f p)$ and an amplitude-modulated $(f a)$ higher frequency. To calculate the MI the phases of phase-modulating oscillation between 0 and $360^{\circ}$ were binned into eighteen $20^{\circ}$ wide bins and the mean amplitude of $f a$ associated to each $20^{\circ}$ wide bin was computed. Then the mean amplitude in each bin was normalized by dividing each bin's value by the sum of all 18 bins, resulting in a phase-amplitude function. A uniform phase-amplitude distribution, that is, the $f a$ amplitude is the same for all $f p$ phase bins, indicates the absence of phase-amplitude coupling. The higher the coupling between $f a$ amplitude and $f p$ phase, the further away from the uniform distribution the phase-amplitude distribution will get. The distance between the two distributions $\mathrm{P}$ and $\mathrm{Q}$ can be defined as $D_{K L}(P, Q)=\sum_{j=1}^{N} P(j) \log \left(\frac{P(j)}{Q(j)}\right)$ where $\mathrm{N}=18$ is the number of phase bins, $\mathrm{j}$ is a number of the given bin (Tort et al., 2008, 2010). The modulation index (MI) is given by $\mathrm{MI}=\frac{D_{K L}(P, Q)}{\log (N)}$ which is a measure between 0 (no phase-amplitude modulation) and 1 (maximal phase-amplitude modulation) (Tort et al., 2008, 2010).

\section{ESTIMATION OF PHASE SYNCHRONIZATION AND COHERENCE}

Coherence between EEG signals was calculated for every patient separately using IMF for which significant CFC was found. For example, when in a particular patient the CFC was found between ACC IMF phase with frequency $3.5 \mathrm{~Hz}$ and AC gamma amplitude with frequency $35 \mathrm{~Hz}$, then single frequency coherence and phase synchronization (PS) were also calculated in this particular patient between all three tested ROI (i.e., ACC, AC, and DLPFC) for the IMF in delta $(3.5 \mathrm{~Hz})$ and gamma $(35 \mathrm{~Hz})$. Coherence was calculated using the standard MATLAB function "mscohere," subsequently averaging across all epochs of a given patient (1.5-s sliding window). To calculate PS continuously over time rather than across trial repetitions, the phase synchronization (Tass et al., 1998; Rosenblum et al., 2001)—also called phase locking value (PLV) (Lachaux et al., 1999)—was defined by PS $=\frac{1}{N} \mid \sum_{n=1}^{N} \exp \left(j \theta\left(\right.\right.$ source $_{x}$, source $\left.\left._{y}, \mathrm{n}\right)\right) \mid$ where $\theta$ $\left(\right.$ source $_{x}$, source $\left._{y}, \mathrm{n}\right)$ is the phase difference between 2 source signals $\phi 1\left(\right.$ source $\left._{x}, \mathrm{n}\right)-\phi 2\left(\right.$ source $\left._{y}, \mathrm{n}\right)$ and $\mathrm{N}$ is the number of time points in the analyzed signal. PS measures the variability of the phase difference between two signals over a certain time interval and reflects the consistency of phase lag between them. To this end, PS detects whether there is one prominent peak in the distribution of the phase difference (Tass et al., 1998; Rosenblum et al., 2001). If the phase difference varies little, PS is close to 1; otherwise it is close to zero.

\section{STATISTICAL ANALYSIS}

In the current study, interactions between changes of MI in PC and NPC groups were investigated using repeated measure analysis of variance (rmANOVA). MIs at the 12 weeks visit were compared to the baseline values using paired samples $t$ test. Comparisons between MIs in tinnitus patients and healthy controls were performed using $t$-tests for independent groups. Pearson correlation coefficients were calculated to study whether the changes of MI correlated with changes in tinnitus pitch change ratio, VAS-L and VAS-A. Pearson correlation was also performed between changes of MI, PS and coherence. In this study we only use the off-stimulation VAS scores, because EEG recordings were performed off-stimulation. The data are presented as mean [standard deviation]. Results of the statistical tests were corrected for the number of tests conducted using the false discovery rate method (FDR) (Benjamini and Hochberg, 1995).

\section{RESULTS}

\section{CROSS-FREQUENCY INTERACTIONS WITHIN AND BETWEEN ROIS}

At the 12 weeks visit VAS-L/VAS-A in all 32 patients were reduced by $43.3 / 44.8 \%$ respectively. In the NPC and PC groups VASL/VAS-A decreased respectively by $44.2 / 43.4 \%$ and $42.4 / 44.2 \%$. In this study we only use the off-stimulation VAS scores, because EEG recordings were performed off-stimulation. Modulus of the pitch change ratio was 0.336 in all 32 patients and $0.151 / 0.521$ in the NPC and PC groups respectively.

As a first step, we compared the levels of hierarchical phaseamplitude coupling in EEG recordings of the tinnitus patients and healthy controls. At baseline, the comodulation analysis revealed the existence of increased (as compared to the healthy controls) phase-amplitude coupling in the tinnitus patients in the AC and DLPFC between the phase of the delta-theta IMFs (mean IMF frequencies for AC: $3.53[0.79] \mathrm{Hz}$ and DLPFC: $3.21[0.90 \mathrm{~Hz}])$ and 
the amplitude of the gamma oscillations (AC: $34.84[2.85]$ and DLPFC: 36.00 [1.59] Hz; Figures 1A,C,D,F, 2A). Decreased CFC was found in tinnitus patients as compared to healthy controls in the AC and DLPFC between the phase of the alpha IMFs (mean IMF frequency for AC: $10.19[0.76]$ and DLPFC: $10.61[0.69] \mathrm{Hz}$ ) and the amplitude of the gamma oscillations (AC: 35.81[2.43] and DLPFC: $36.20[3.39] \mathrm{Hz}$; Figures 1G,I,J,L, 2B). At the 12week follow up these CFCs in tinnitus patients were significantly modified and approached the levels of the control group (Figures 1, 2A,B).

Further we investigated CFC between phases of the lower frequency modes (delta, theta and alpha) in ACC and amplitudes of the gamma oscillations in the AC and DLPFC. CFC in tinnitus patients as compared to the healthy controls was increased between ACC theta phase (IMF frequency $5.85[0.76] \mathrm{Hz}$ ) and the amplitude of the gamma oscillation in the DLPFC (IMF frequency $36.67[2.54] \mathrm{Hz}$ ) and AC (IMF frequency $36.61[2.0] \mathrm{Hz}$; Figures 2C, 3A,C,D,F). This CFC averaged over all of the 32 patients was significantly reduced at the 12-week follow up, as compared to the baseline, between the ACC and DLPFC (MI change $-0.022, p<0.001)$ and between the ACC and AC (MI change $-0.062, p<0.001$; Figures 3A-F).

We next investigated whether there was an interaction between MI changes after 12 weeks of CR neuromodulation in the PC and NPC groups. The rmANOVA revealed a significant interaction $(p<0.05)$ between ACC theta-DLPFC gamma MI changes in the PC and NPC groups. Specifically, ACC thetaDLPFC gamma MI reduced significantly greater in the PC group

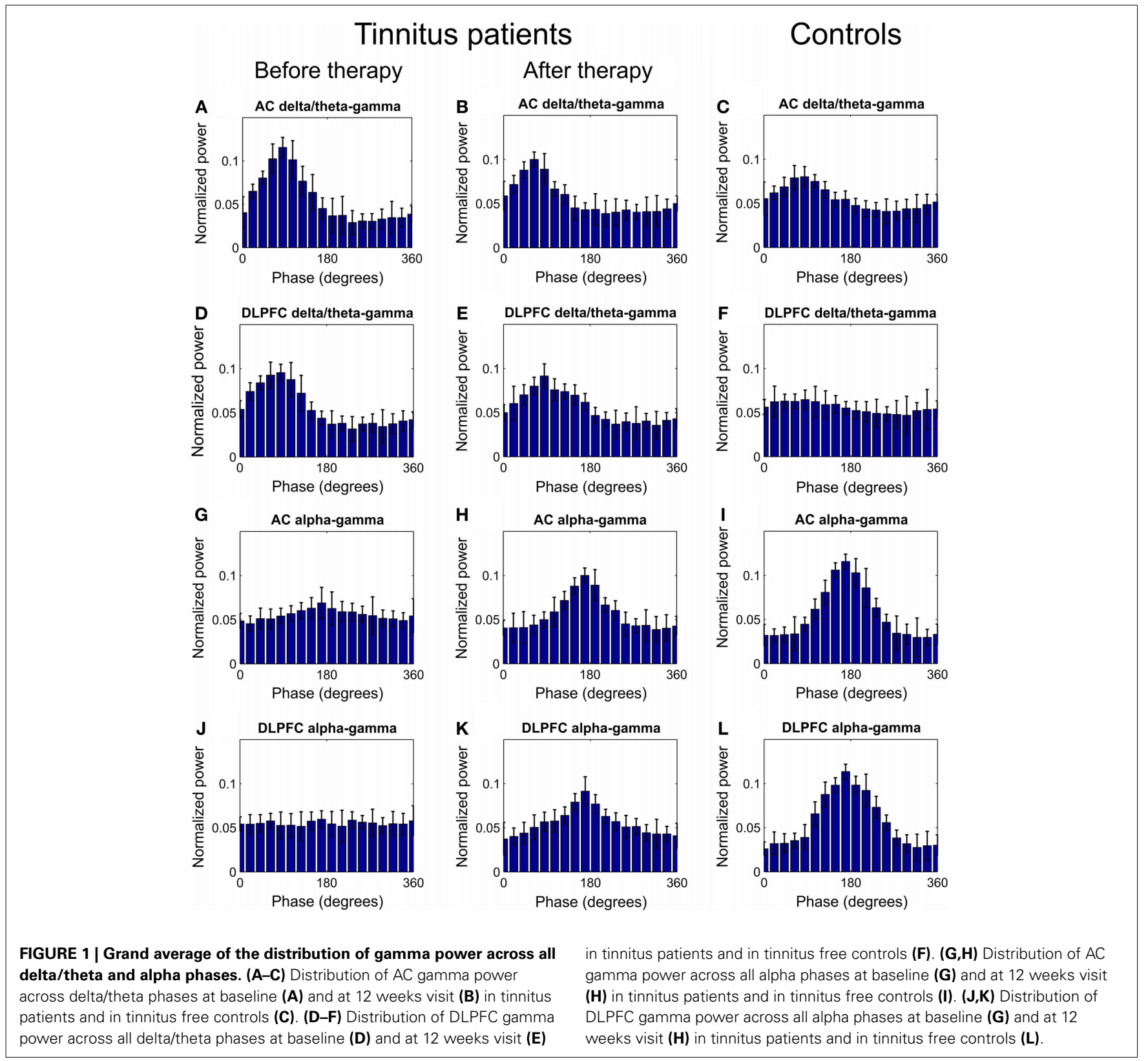



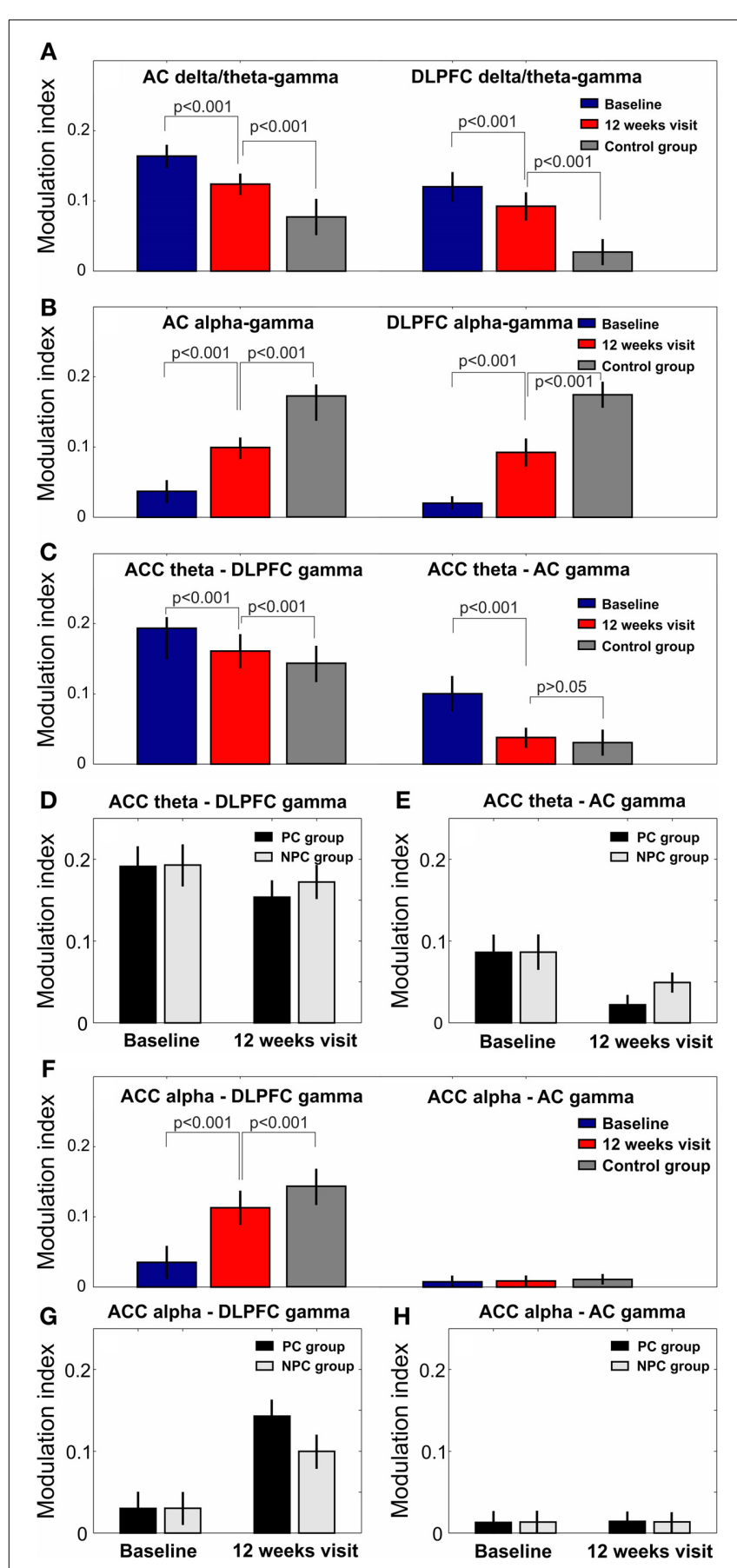

FIGURE 2 | The modulation index values in all $n=32$ investigated patients at baseline and at the 12 weeks visit. The gamma amplitude delta/theta phase modulation index in the AC and DLPFC (A). The gamma amplitude alpha phase modulation index in the AC and DLPFC (B). After 12 weeks of acoustic CR neuromodulation the CFC between delta/theta phase and gamma amplitude approached the CFC in the healthy control group (A,B). CFC between ACC theta phase and DLPFC/AC gamma amplitude (C). The modulation index values for CFC between ACC theta phase and DLPFC/AC gamma amplitude in both PC $(n=16)$ and NPC $(n=16)$ groups at baseline and at 12 weeks visit $(\mathbf{D}, \mathbf{E})$. The reduction of CFC between ACC theta phase and DLPFC/AC gamma amplitude was significantly more pronounced in the PC group than in the NPC group at 12 weeks visit

(Continued)

\section{FIGURE 2 | Continued}

as compared to baseline (D,E). CFC between ACC alpha phase and DLPFC/AC gamma amplitude (F). The increase of CFC between ACC alpha phase and DLPFC gamma amplitude was significantly more pronounced in the PC group than in the NPC group at 12 weeks visit as compared to baseline $(\mathbf{G}, \mathbf{H})$.

(MI change $-0.033, p<0.001)$ as compared to the NPC group (MI change $-0.010, p<0.001$; Figure 2D). Significant interaction was also revealed for the ACC theta-AC gamma CFC with respect to group $(p<0.05)$. The reduction of $\mathrm{MI}$ in the $\mathrm{PC}$ group was significantly more pronounced (MI change -0.074 , $p<0.001$ ) as compared to the NPC group (MI change -0.050 , $p<0.001$; Figure 2E).

At baseline there was a decreased CFC between the ACC alpha phase and the amplitude of the gamma oscillations in the DLPFC (Figures 2F, 3G,I) but not between the ACC alpha and the AC gamma (Figures 2F, 3J,L) in tinnitus patients as compared to the healthy controls. At the 12 weeks follow up, these CFCs averaged over all of the 32 tinnitus patients were MI 0.120 (MI change $0.085, p<0.001$ ) between the ACC and the DLPFC (Figures 2F, 3H) and MI 0.009 (MI change 0.001, $p>0.05$ ) between the ACC and AC (Figures 2F, 3K). The rmANOVA revealed a significant interaction $(p<0.05)$ between MI changes with respect to group, that is, ACC alpha-DLPFC gamma phase-amplitude coupling increased significantly more pronounced in the PC group (MI change 0.106, $p<0.001$ ) as compared to the NPC group (MI change 0.064, $p<0.001$; Figure 2G).

Changes of CFC significantly correlated with changes of VAS-L/VAS-A and modulus of tinnitus pitch change (Table 1). Changes of theta-gamma CFC between ACC and DLPFC as well as between ACC and AC were qualitatively similar as revealed by strong significant correlation between ACC theta-DLPFC gamma and ACC theta-AC gamma MI changes $(r=0.67$, $p<0.01)$. These results together with absence of significant changes of the phase synchronization between AC and DLPFC (Tables 2, 3) indicate that the gamma amplitude in the AC and DLPFC may have been similarly modulated by the theta phase in the ACC.

\section{PHASE SYNCHRONIZATION AND COHERENCE BETWEEN ROIS}

To further investigate the processes that lead to abnormal crossfrequency interactions in tinnitus patients and CFC changes after the CR therapy, we analyzed the PS and coherence at baseline and at 12 weeks as well as PS and coherence changes between baseline and 12 weeks for all subjects. We focused on the same frequency PS and coherence between pairs of ROIs, i.e., interareal interactions within delta, theta, alpha and gamma bands. The results of the phase synchronization and coherence analysis are shown in Tables 2, 3. Significant changes of the PS and coherence were observed only in the gamma frequency band between $\mathrm{AC}$ and DLPFC (Tables 2, 3). No significant correlation was found between the changes of the PS or coherence in any of the frequency bands and changes of CFC. What is noteworthy here is the fact that the PS 


\section{Tinnitus patients}

Before therapy
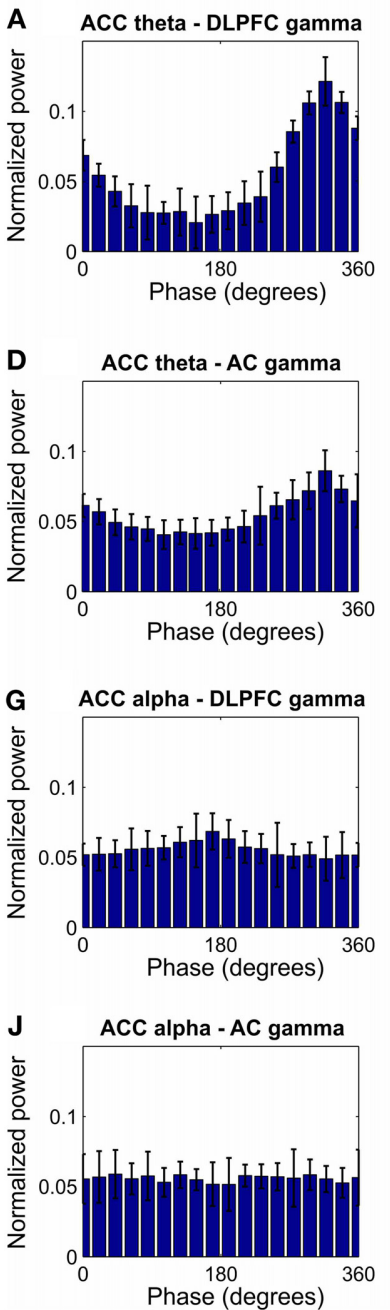

After therapy
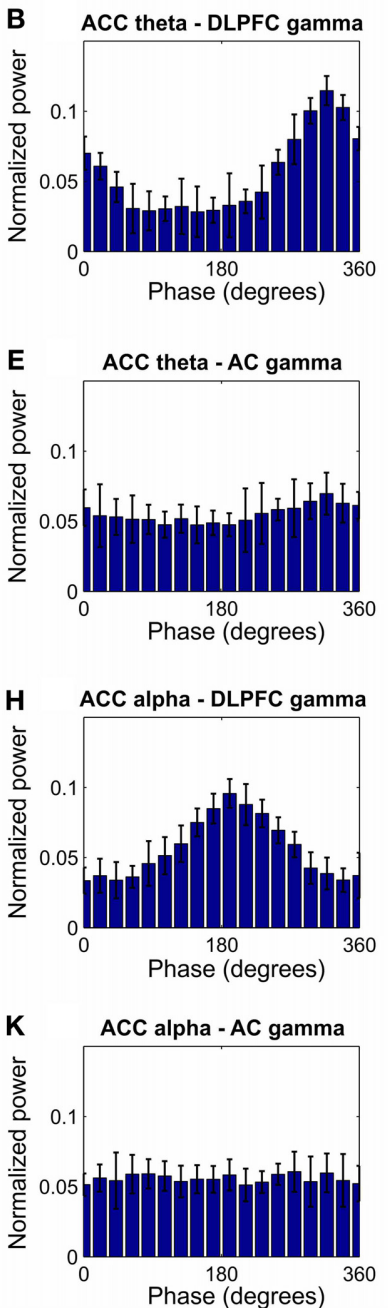
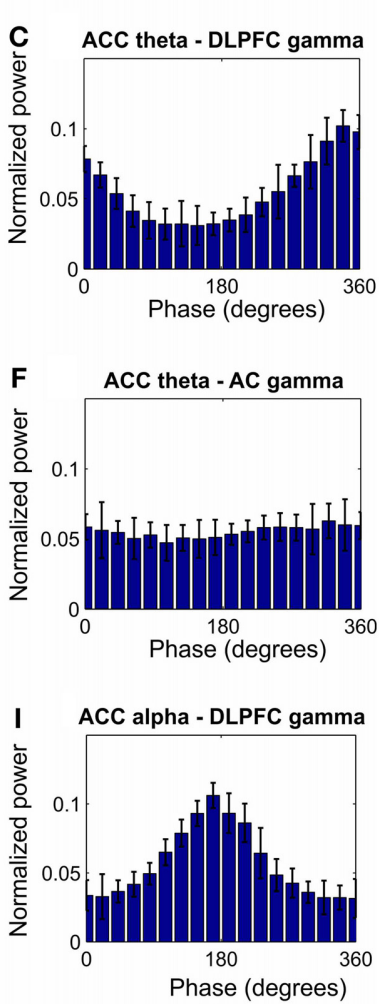

\section{Controls}

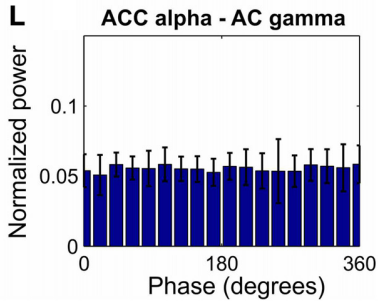

FIGURE 3 | Grand average of the distribution of gamma power across all ACC theta and alpha phases. (A-C) Distribution of DLPFC gamma power across all theta phases of ACC theta at baseline (A) and at 12 weeks visit (B) in tinnitus patients and in tinnitus free controls (C). (D-F) Distribution of AC gamma power across all theta phases of ACC theta at baseline (D) and at 12 weeks visit (E) in

Table 1 | Correlations between changes of MI and changes of the modulus of the tinnitus pitch change ratio, VAS-L/VAS-A.

Modulus of relative pitch change

$$
\begin{gathered}
r=-0.41, p=0.03 \\
r=-0.40, p=0.02 \\
r=0.66, p<0.01
\end{gathered}
$$

Relative VAS-L change

Relative VAS-A change

MI change ACC theta-AC gamma
MI change ACC theta-DLPFC gamma
MI change ACC theta-DLPFC gamma

and coherence changes between areas (e.g., between ACC and DLPFC) within modulating frequencies (delta, theta and alpha) that were involved in the intraareal cross-frequency modulation of the gamma band were not correlated with the CFC tinnitus patients and in tinnitus free controls (F). (G,H) Distribution of DLPFC gamma power across all ACC alpha phases at baseline (G) and at 12 weeks visit (H) in tinnitus patients and in tinnitus free controls (I). (J-L) Distribution of AC gamma power across all alpha phases in ACC at baseline (G) and at 12 weeks visit $(\mathbf{H})$ in tinnitus patients and in tinnitus free controls (L) 
Table 2 | Coherence between selected regions for the selected frequency bands.

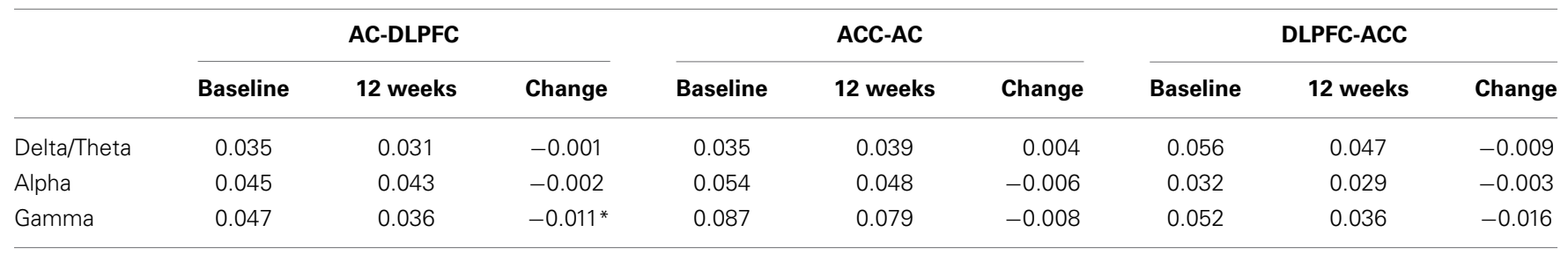

The baseline and 12 weeks coherence and the change during the 12 weeks treatment period are shown for the bands where CFC interaction was found. ${ }^{*} p<0.05, n=32$.

Table 3 | Phase synchronization between selected regions for the selected frequency bands.

\begin{tabular}{|c|c|c|c|c|c|c|c|c|c|}
\hline & \multicolumn{3}{|c|}{ AC-DLPFC } & \multicolumn{3}{|c|}{ ACC-AC } & \multicolumn{3}{|c|}{ DLPFC-ACC } \\
\hline & Baseline & 12 weeks & Change & Baseline & 12 weeks & Change & Baseline & 12 weeks & Change \\
\hline Alpha & 0.16 & 0.20 & 0.04 & 0.16 & 0.17 & 0.02 & 0.16 & 0.20 & 0.04 \\
\hline Gamma & 0.18 & 0.11 & $-0.07^{*}$ & 0.18 & 0.17 & -0.02 & 0.18 & 0.12 & -0.06 \\
\hline
\end{tabular}

${ }^{*} p<0.05, n=32$.

\section{DISCUSSION}

Both animal (Eggermont and Komiya, 2000; Eggermont, 2003; Seki and Eggermont, 2003) and human data (Weisz et al., 2005; van der Loo et al., 2009; De Ridder et al., 2011b; Adjamian et al., 2012; Adamchic et al., 2014) demonstrate that tinnitus is associated with increased oscillatory activity in delta, theta and gamma bands and increased neuronal synchronization in the auditory cortex and that the more this increase of neuronal activity and synchronization is pronounced, the higher is the intensity of the perceived tinnitus. Furthermore, reduction of oscillatory power in the delta/theta and gamma frequency ranges and increase of the oscillatory power in the alpha band in this area is associated with reduction of tinnitus loudness (Kahlbrock and Weisz, 2008; De Ridder et al., 2011b; Adjamian et al., 2012; Tass et al., 2012; Adamchic et al., 2014). The thalamocortical dysrhythmia model provides an explanation for the emergence and persistence of such a pattern of oscillatory activity as a result of sensory differentiation (Llinas et al., 1999). Particularly this model suggests that gamma oscillations emerge together with an increase in slow wave activity (Llinas et al., 1999; Weisz et al., 2007). This notion is further confirmed and extended by the current study, which revealed that in tinnitus, aside from the described changes of oscillatory activity, there is also a more pronounced periodicity of amplitude variations of higher frequency (gamma) oscillations locked to certain phases of lower (delta/theta) frequency oscillations. Moreover reduction of initially increased cross-frequency interaction in the tinnitus patients was positively correlated with reduction of tinnitus after treatment with $\mathrm{CR}$ neuromodulation.

In our previous publications (Tass et al., 2012; Adamchic et al., 2014) and in the current study we revealed that in tinnitus both sensory and prefrontal areas are affected by similar abnormalities of oscillatory power and CFC. Moreover, the amount of the therapy induced pitch change was associated with the amount of changes of these abnormalities (Adamchic et al., 2012a).
According to these results and the findings of previous studies (Weisz et al., 2005, 2006, 2007; Schlee et al., 2009; Vanneste et al., 2011; Adamchic et al., 2012a, 2014; Eggermont and Roberts, 2012; Tass et al., 2012), we can summarize that conscious tinnitus perception involves abnormalities in activity of auditory cortices together with abnormal processing across cortical circuits, including the prefrontal cortex. Moreover, the oscillatory activity in both the sensory and prefrontal regions may be modulated by other cortical areas in a tinnitus aversive network, specifically, the limbic system (Jastreboff, 1990; Rauschecker et al., 2010; De Ridder et al., 2011a, 2014). In such a network, coupled delta/theta-gamma activity may serve as an important mechanism for communication between these areas (Canolty et al., 2006).

In modeling studies it was shown that periodically firing or bursting neurons display the dynamics of limit cycle oscillators (Murray, 1989) which can be approximated by means of phase oscillators (Kuramoto, 1984; Hansel et al., 1993a,b). Mathematically it was shown that the coupling between neurons may cause mutual phase differences, frequency shifts, different types of synchronization patterns such as in-phase synchrony and cluster states (Tass, 1995, 1997). For strong enough coupling stable fixpoint-type states emerge, whereas for weaker coupling socalled running solutions emerge, where these different variables undergo variations in time (Murray, 1989; Guckenheimer and Holmes, 1990). On mesoscopic or macroscopic levels, assessed by local field potentials or MEG/EEG recordings, this translates to a variety of modulatory dynamical phenomena, such as power to power CFC, phase to phase CFC, phase to power CFC, phase to frequency CFC, power to frequency CFC, and frequency to frequency CFC (for review see Jirsa and Müller, 2013). Transient coupling between low and high frequencies was proposed to coordinate activity between task-relevant regions providing a mechanism for effective communication during cognitive, sensory and memory processing (Buzsaki and Draguhn, 2004; Canolty et al., 
2006; Doesburg et al., 2012). Brain oscillations of different frequencies can interact within and between various brain areas, e.g., in the following ways: (i) amplitude modulation of a higher frequency oscillation by the phase of a slower rhythm, that is, phase-amplitude coupling (ii) phase modulation of a higher frequency oscillation by the phase of a slower rhythm, that is, phase-phase coupling (iii); amplitude modulation of a higher frequency oscillation by the amplitude of a slower rhythm that is, amplitude-amplitude coupling (Tass et al., 1998; Payne and Kounios, 2009; Canolty and Knight, 2010; Tort et al., 2010; Fell and Axmacher, 2011; Onslow et al., 2011). These types of CFC may occur independently, may have different mechanisms and can lead to different functional consequences (Fell and Axmacher, 2011). Phase-amplitude coupling was demonstrated in working memory tasks in which rats, while navigating through a T-maze, had to make decisions and thus recruit working or long-term memory (Tort et al., 2008). In humans phase-amplitude coupling between theta phase and gamma $(20-40 \mathrm{~Hz})$ frequency oscillations amplitude increased while retaining novel information in the working memory (Axmacher et al., 2010). Based on these findings it has been suggested that phase-amplitude coupling mechanisms in the limbic system and prefrontal cortex may support phase-dependent coding of objects (Fell and Axmacher, 2011).

Our study revealed that alpha oscillations exert crossfrequency influence on gamma activity. Alpha oscillations were proposed to exercise "pulsed" functional inhibition of irrelevant neuronal processing streams (Jensen and Mazaheri, 2010; Mazaheri and Jensen, 2010; Siegel et al., 2012). Accordingly, in the tinnitus network transient periods of alpha-entrainment from the ACC may pulse modulate gamma activity (Osipova et al., 2008) in the DLPFC as implied by the increase of alpha activity in the ACC, increase of phase-amplitude CFC (between the ACC and the DLPFC) and the simultaneous decrease of gamma power in the DLPFC with increase of tinnitus pitch change (Adamchic et al., 2012a). Thus, we can speculate that increase in the alpha activity in the ACC through its influence on an inhibitory control system of the dorsolateral prefrontal cortex can lead to an increase in the magnitude of inhibitory bouts on the DLPFC and break the local ongoing gamma activity (Medalla and Barbas, 2009; Jensen and Mazaheri, 2010; Mazaheri and Jensen, 2010). This notion would be in line with data showing that the ACC has strong influence on an inhibitory control system of the dorsolateral prefrontal cortex, more specifically BA 9, and can effectively enhance inhibition in that area (Medalla and Barbas, 2009). The stronger ACC alpha may lead to the shorter "duty-cycle" of gamma activity in the DLPFC providing graded inhibition of gamma oscillations by blocking it in a phasic manner, that is, by decreasing the time-window (duty-cycle) of processing (Jensen and Mazaheri, 2010). Reversing this, the reduction of alpha oscillations in patients with chronic tinnitus may lengthen the time-windows for gamma activity, thus, leading to increased gamma activity. Coupling of alpha oscillation with higher frequency, that is, gamma neural activity, may be ubiquitous throughout the cortex and thus throughout the tinnitus network (Palva et al., 2005, 2010; Palva and Palva, 2007; Osipova et al., 2008; Voytek et al., 2010). This notion is further supported by our finding of alpha-gamma CFC within the AC and the DLPFC.

Involvement of theta activity in tinnitus pathophysiology is also widely accepted (Llinas et al., 1999; De Ridder et al., 2011b). In our previous studies we have shown that tinnitus reduction by CR neuromodulation is related to a normalization of increased theta activity in the medial prefrontal cortex (Tass et al., 2012; Adamchic et al., 2014). The modulation of gamma-band synchronization by the theta rhythm was established in multiple studies (Bragin et al., 1995; Lakatos et al., 2005; Canolty et al., 2006). The phase of the theta oscillation may define the time window when transfer of information is most efficacious (Hasselmo et al., 2002a,b; Fell and Axmacher, 2011). Moreover, oscillations in the theta frequency range may be more efficacious for information transfer over longer distances in the brain than gamma phase synchronization (von Stein and Sarnthein, 2000; Hasselmo et al., 2002a,b; Buzsaki, 2009). Thus, within the tinnitus network theta activity may coordinate local assemblies that are synchronized in the gamma range. Our analysis revealed that the phase of theta activity in the ACC modulated the synchronization in the gamma band in both the AC and the DLPFC. Increased interaction between the DLPFC and sensory lower-order areas, that is, the $\mathrm{AC}$ (that is confirmed by the significant reduction of the phase synchronization between DLPFC and AC) during presence of aversive auditory pitch may serve the purpose to prioritize further processing of that stimulus as the most relevant (Zatorre et al., 1994; Fuster, 2008). Such modulation of oscillatory rhythms in both the AC and the DLPFC regions appears to be coordinated by the ACC, and more specifically by the theta rhythm. Thus, coordinated theta activity may establish long-range brain networks, in which information is processed by gamma activity, nested on this theta activity. The ACC may have an important role in regulation of neuronal circuitry for cognitive and sensory domains and can be identified as a distinctive region in the tinnitus affective network.

For the within-band interaction, we did not find consistent changes of phase synchronization or coherence between the investigated anatomical regions (i.e., between DLPFC and ACC and between ACC and AC) in the frequency bands that were involved in the phase-amplitude CFC, except reduction of the gamma PS and coherence between AC and DLPFC. In addition, the values of PS are, in general, small. This shows, on the one hand, that the observed CFC is not an epiphenomenon of an underlying single band synchronization between the two regions. On the other hand, because theoretically single-band synchronization and CFC between the same two regions can coexist, the phaseamplitude CFC shown here is an exclusively non-linear effect independent from the within-band synchronization in the frequency band involved in the phase-amplitude CFC between the two involved regions (Darvas et al., 2009; López-Azcárate et al., 2013). Furthermore, the independence between changes of CFC and single-band phase synchronization changes allow to speculate that the observed CFC in the tinnitus network may provide an information transfer between nodes of this network not realizable by simple single-band synchronization.

An alternative explanation for CFC without any apparent interareal phase synchronization in the e.g., theta band is that 
feed-forward inhibition rather than excitation may be the main contributor to the observed CFC. Specifically, phase locking to the modulating frequency in the modulated area may arise through modulation of pyramidal cells excitability mediated via local inhibitory networks (Tierney et al., 2004; Siapas et al., 2005). This may explain the modulation of auditory and prefrontal gamma activity by limbic theta activity even in the apparent absence of phase synchronous theta activity in the modulated regions (i.e., prefrontal and auditory cortices), and as a result absence of any apparent interareal phase synchronization. If the output of the cingulate cortex, also through polysynaptic connections, terminates not on the pyramidal prefrontal and auditory neurons but rather on interneurons, then generated rhythmic synaptic currents are unlikely to add to a microscopic field oscillation (Niedermeyer and Lopes da Silva, 1999; Siapas et al., 2005) because of rather random geometric configuration and small size of the interneurons and their dendrites. Thus, rather than hyperpolarizing the pyramidal cells, the cingulate input can rhythmically decrease pyramidal cells impedance, thus modulating their excitability (Niedermeyer and Lopes da Silva, 1999; Siapas et al., 2005). Another, more general explanation for the amplitude of a fast rhythm being modulated by the phase of a slow rhythm comes from a computational study showing that periodic input into generic networks of bursting neurons or networks of phase oscillators causes a quasiperiodic modulation of the amplitude of the macroscopic variable assessing the network's collective dynamics (i.e., the local field potential for the bursting neurons) over a wide range of the input intensity (see Figures 2, 7 in Popovych and Tass, 2011). Only at particularly high input intensities an n:m phase synchronization emerges (Popovych and Tass, 2011). Put otherwise, from a modeling standpoint this phenomenon actually occurs in a generic way for biologically realistic, i.e., weak to intermediate input intensities.

In our current study we found clear evidence of the influence of tinnitus severity and tinnitus pitch on theta-gamma and alpha-gamma coupling. More specifically, CFC between theta in the ACC and gamma in the DLPFC and the AC was increased in tinnitus patients and was reduced after reduction of tinnitus severity by CR therapy. Similarly tinnitus pitch reduction after therapy resulted in a decrease of the theta-gamma and increase of the alpha-gamma coupling. Thus, the strength of cross-frequency modulation of cognitive and sensory areas by limbic system may be related to the perceived subjective aversiveness of the tinnitus, which in turn depends on both loudness and pitch.

\section{CONCLUSION}

The current study showed that reduction of tinnitus severity had a large impact on the cross-frequency interaction in the AC, the DLPFC as well as between the ACC and both the DLPFC and the AC. Even though the reduction of CFC was not solely reliant on the amount of tinnitus pitch change, the presence of pronounced tinnitus pitch change significantly increased changes in CFC. Modulation of the amplitude of high-frequency oscillations by the phase of oscillations in lower frequency ranges can coordinate tinnitus-relevant activity in distributed cortical areas providing a mechanism for effective communication between these areas during cognitive, memory, and auditory processing of the tinnitus, that is, orchestrating a widespread tinnitus-related gamma network. The strength of the interaction between cognitive, sensory areas and the limbic system may reflect both the tinnitus related distress and the subjective aversion to the perceived auditory pitch.

\section{REFERENCES}

Aazh, H., Moore, B. C., and Glasberg, B. R. (2008). Simplified form of tinnitus retraining therapy in adults: a retrospective study. BMC Ear. Nose Throat. Disord. 8:7. doi: 10.1186/1472-6815-8-7

Adamchic, I., Hauptmann, C., and Tass, P. A. (2012a). Changes of oscillatory activity in pitch processing network and related tinnitus relief induced by acoustic CR neuromodulation. Front. Syst. Neurosci. 6:18. doi: 10.3389/fnsys.2012. 00018

Adamchic, I., Langguth, B., Hauptmann, C., and Tass, P. A. (2012b). Psychometric evaluation of Visual Analog Scale for the assessment of chronic tinnitus. Am. J. Audiol. 21, 215-225. doi: 10.1044/1059-0889(2012/12-0010)

Adamchic, I., Tass, P. A., Langguth, B., Hauptmann, C., Koller, M., Schecklmann, M., et al. (2012c). Linking the tinnitus questionnaire and the subjective clinical global impression: which differences are clinically important? Health Qual. Life Outcomes 10:79. doi: 10.1186/1477-7525-10-79

Adamchic, I., Toth, T., Hauptmann, C., and Tass, P. A. (2014). Reversing pathologically increased EEG power by acoustic coordinated reset neuromodulation. Hum. Brain Mapp. 35, 2099-2118. doi: 10.1002/hbm.22314

Adjamian, P., Sereda, M., Zobay, O., Hall, D. A., and Palmer, A. R. (2012). Neuromagnetic Indicators of Tinnitus and Tinnitus Masking in Patients with and without Hearing Loss. J. Assoc. Res. Otolaryngol. 13, 715-731. doi: 10.1007/s10162-012-0340-5

Axmacher, N., Henseler, M. M., Jensen, O., Weinreich, I., Elger, C. E., and Fell, J. (2010). Cross-frequency coupling supports multi-item working memory in the human hippocampus. Proc. Natl. Acad. Sci. U.S.A. 107, 3228-3233. doi: 10.1073/pnas.0911531107

Bartels, H., Staal, M. J., Holm, A. F., Mooij, J. J., and Albers, F. W. (2007). Long-term evaluation of treatment of chronic, therapeutically refractory tinnitus by neurostimulation. Stereotact. Funct. Neurosurg. 85, 150-157. doi: 10.1159/000099073

Benjamini, Y., and Hochberg, Y. (1995). Controlling the false discovery rate: a practical and powerful approach to multiple testing. J. R. Stat. Soc. Ser. B 57, 289-300.

Bragin, A., Jando, G., Nadasdy, Z., Hetke, J., Wise, K., and Buzsaki, G. (1995). Gamma $(40-100-\mathrm{Hz})$ Oscillation in the hippocampus of the behaving rat. J. Neurosci. 15, 47-60.

Buzsaki, G. (2009). Rhythms of the Brain. Oxford University Press.

Buzsaki, G., and Draguhn, A. (2004). Neuronal oscillations in cortical networks. Science 304, 1926-1929. doi: 10.1126/science.1099745

Canolty, R. T., Edwards, E., Dalal, S. S., Soltani, M., Nagarajan, S. S., Kirsch, H. E., et al. (2006). High gamma power is phase-locked to theta oscillations in human neocortex. Science 313, 1626-1628. doi: 10.1126/science.1128115

Canolty, R. T., and Knight, R. T. (2010). The functional role of cross-frequency coupling. Trends Cogn. Sci. 14, 506-515. doi: 10.1016/j.tics.2010.09.001

Darvas, F., Miller, K. J., Rao, R. P., and Ojemann, J. G. (2009). Nonlinear phasephase cross-frequency coupling mediates communication between distant sites in human neocortex. J. Neurosci. 29, 426-435. doi: 10.1523/JNEUROSCI.368808.2009

De Ridder, D., Elgoyhen, A. B., Romo, R., and Langguth, B. (2011a) Phantom percepts: tinnitus and pain as persisting aversive memory networks. Proc. Natl. Acad. Sci. U.S.A. 108, 8075-8080. doi: 10.1073/pnas.10184 66108

De Ridder, D., van der Loo, E., Vanneste, S., Gais, S., Plazier, M., Kovacs, S., et al. (2011b). Theta-gamma dysrhythmia and auditory phantom perception. J. Neurosurg. 114, 912-921. doi: 10.3171/2010.11.JNS10335

De Ridder, D., and Vanneste, S. (2011). "Auditory cortex stimulation for tinnitus," in Textbook of Tinnitus, eds A. R. Moller, B. Langguth, D. De Ridder, and T. Kleinjung (New York, NY: Springer), 717-726.

De Ridder, D., Vanneste, S., Weisz, N., Londero, A., Schlee, W., Elgoyhen, A. B., et al. (2014). An integrative model of auditory phantom perception: tinnitus as a unified percept of interacting separable subnetworks. Neurosci. Biobehav. Rev. 44, 16-32. doi: 10.1016/j.neubiorev.2013.03.021 
Doesburg, S. M., Green, J. J., McDonald, J. J., and Ward, L. M. (2012). Theta modulation of inter-regional gamma synchronization during auditory attention control. Brain Res. 1431, 77-85. doi: 10.1016/j.brainres.2011.11.005

Eggermont, J. J. (2003). Central tinnitus. Auris Nasus Larynx 30(Suppl. ), S7-S12. doi: 10.1016/S0385-8146(02)00122-0

Eggermont, J. J., and Komiya, H. (2000). Moderate noise trauma in juvenile cats results in profound cortical topographic map changes in adulthood. Hear. Res. 142, 89-101. doi: 10.1016/S0378-5955(00)00024-1

Eggermont, J. J., and Roberts, L. E. (2012). The neuroscience of tinnitus: understanding abnormal and normal auditory perception. Front. Syst. Neurosci. 6:53. doi: $10.3389 /$ fnsys. 2012.00053

Fell, J., and Axmacher, N. (2011). The role of phase synchronization in memory processes. Nat. Rev. Neurosci. 12, 105-118. doi: 10.1038/nrn2979

Florin, E., Gross, J., Pfeifer, J., Fink, G. R., and Timmermann, L. (2010). The effect of filtering on Granger causality based multivariate causality measures. Neuroimage 50, 577-588. doi: 10.1016/j.neuroimage.2009.12.050

Fuster, J. (2008). The Prefrontal Cortex. London: Academic Press.

Guckenheimer, J., and Holmes, P. (1990). Nonlinear Oscillations, Dynamical Systems, and Bifurcations of Vector Fields. Springer.

Hansel, D., Mato, G., and Meunier, C. (1993a). Phase dynamics for weakly coupled hodgkin-huxley neurons. Europhys. Lett. 23, 367-372. doi: 10.1209/0295$5075 / 23 / 5 / 011$

Hansel, D., Mato, G., and Meunier, C. (1993b). Phase reduction and neural modeling. Concepts Neurosci. 4, 193-210.

Hasselmo, M. E., Bodelon, C., and Wyble, B. P. (2002a). A proposed function for hippocampal theta rhythm: separate phases of encoding and retrieval enhance reversal of prior learning. Neural Comput. 14, 793-817. doi: $10.1162 / 089976602317318965$

Hasselmo, M. E., Hay, J., Ilyn, M., and Gorchetchnikov, A. (2002b). Neuromodulation, theta rhythm and rat spatial navigation. Neural Netw. 15, 689-707. doi: 10.1016/S0893-6080(02)00057-6

Huang, N. E., Shen, Z., Long, S. R., Wu, M. L. C., Shih, H. H., Zheng, Q. N., et al. (1998). The empirical mode decomposition and the Hilbert spectrum for nonlinear and non-stationary time series analysis. Proc. R. Soc. Lond. Ser. Math. Phys. Eng. Sci. 454, 903-995. doi: 10.1098/rspa.1998.0193

Ille, N., Berg, P., and Scherg, M. (2002). Artifact correction of the ongoing EEG using spatial filters based on artifact and brain signal topographies. J. Clin. Neurophysiol. 19, 113-124. doi: 10.1097/00004691-20020300000002

Jastreboff, P. J. (1990). Phantom auditory perception (tinnitus): mechanisms of generation and perception. Neurosci. Res. 8, 221-254. doi: 10.1016/01680102(90)90031-9

Jensen, O., and Mazaheri, A. (2010). Shaping functional architecture by oscillatory alpha activity: gating by inhibition. Front. Hum. Neurosci. 4:186 doi 10.3389/fnhum.2010.00186

Jirsa, V., and Müller, V. (2013). Cross-frequency coupling in real and virtual brain networks. Front. Comput. Neurosci. 7:78. doi: 10.3389/fncom.2013.00078

Kahlbrock, N., and Weisz, N. (2008). Transient reduction of tinnitus intensity is marked by concomitant reductions of delta band power. BMC Biol. 16:4. doi: 10.1186/1741-7007-6-4

Kuramoto, Y. (1984). Chemical Oscillations, Waves, and Turbulence. Berlin: Springer.

Lachaux, J. P., Rodriguez, E., Martinerie, J., and Varela, F. J. (1999). Measuring phase synchrony in brain signals. Hum. Brain Mapp. 8, 194-208.

Lakatos, P., Shah, A. S., Knuth, K. H., Ulbert, I., Karmos, G., and Schroeder, C. E. (2005). An oscillatory hierarchy controlling neuronal excitability and stimulus processing in the auditory cortex. J. Neurophysiol. 94, 1904-1911. doi: 10.1152/jn.00263.2005

Landgrebe, M., Azevedo, A., Baguley, D., Bauer, C., Cacace, A., Coelho, C., et al. (2012). Methodological aspects of clinical trials in tinnitus: a proposal for an international standard. J. Psychosom. Res. 73, 112-121. doi: 10.1016/j.jpsychores.2012.05.002

Langguth, B., Kreuzer, P. M., Kleinjung, T., and De Ridder, D. (2013). Tinnitus: causes and clinical management. Lancet Neurol. 12, 920-930. doi: 10.1016/S1474-4422(13)70160-1

Llinas, R. R., Ribary, U., Jeanmonod, D., Kronberg, E., and Mitra, P. P. (1999). Thalamocortical dysrhythmia: a neurological and neuropsychiatric syndrome characterized by magnetoencephalography. Proc. Natl. Acad. Sci. U.S.A. 96, 15222-15227. doi: 10.1073/pnas.96.26.15222
López-Azcárate, J., Nicolás, M. J., Cordon, I., Alegre, M., Valencia, M., and Artieda, J. (2013). Delta-mediated cross-frequency coupling organizes oscillatory activity across the rat cortico-basal ganglia network. Front. Neural Circuits. 7:155. doi: 10.3389/fncir.2013.00155

Mazaheri, A., and Jensen, O. (2010). Rhythmic pulsing: linking ongoing brain activity with evoked responses. Front. Hum. Neurosci. 4:177. doi: 10.3389/fnhum.2010.00177

Medalla, M., and Barbas, H. (2009). Synapses with inhibitory neurons differentiate anterior cingulate from dorsolateral prefrontal pathways associated with cognitive control. Neuron 61, 609-620. doi: 10.1016/j.neuron.2009.01.006

Møller, A. R., Langguth, B., Kleinjung, T., and Ridder, D. D. (2010). Textbook of Tinnitus. New York; Dordrecht; Heidelberg; London: Springer.

Mormann, F., Fell, J., Axmacher, N., Weber, B., Lehnertz, K., Elger, C. E., et al. (2005). Phase/amplitude reset and theta-gamma interaction in the human medial temporal lobe during a continuous word recognition memory task. Hippocampus 15, 890-900. doi: 10.1002/hipo.20117

Murray, J. D. (1989). Mathematical Biology. Berlin: Springer-Verlag.

Niedermeyer, E., and Lopes da Silva, F. H. (1999). Electroencephalography. Basic Principals, Clinical Applications, and Related Fields. 4th Edn. London: Williams \& Wilkins.

Onslow, A. C. E., Bogacz, R., and Jones, M. W. (2011). Quantifying phaseamplitude coupling in neuronal network oscillations. Prog. Biophys. Mol. Biol. 105, 49-57. doi: 10.1016/j.pbiomolbio.2010.09.007

Osipova, D., Hermes, D., and Jensen, O. (2008). Gamma power is phase-locked to posterior alpha activity. PLoS ONE 3:e3990. doi: 10.1371/journal.pone.0003990

Palva, J. M., Monto, S., Kulashekhar, S., and Palva, S. (2010). Neuronal synchrony reveals working memory networks and predicts individual memory capacity. Proc. Natl. Acad. Sci. U.S.A. 107, 7580-7585. doi: 10.1073/pnas.0913113107

Palva, J. M., Palva, S., and Kaila, K. (2005). Phase synchrony among neuronal oscillations in the human cortex. J. Neurosci. 25, 3962-3972. doi: 10.1523/JNEUROSCI.4250-04.2005

Palva, S., and Palva, J. M. (2007). New vistas for alpha-frequency band oscillations. Trends Neurosci. 30, 150-158. doi: 10.1016/j.tins.2007.02.001

Payne, L., and Kounios, J. (2009). Coherent oscillatory networks supporting short-term memory retention. Brain Res. 1247, 126-132. doi: 10.1016/j.brainres.2008.09.095

Popovych, O. V., and Tass, P. A. (2011). Macroscopic entrainment of periodically forced oscillatory ensembles. Prog. Biophys. Mol. Biol. 105, 98-108. doi: 10.1016/j.pbiomolbio.2010.09.018

Rauschecker, J. P., Leaver, A. M., and Mühlau, M. (2010). Tuning out the noise: limbic-auditory interactions in tinnitus. Neuron 66, 819-826. doi: 10.1016/j.neuron.2010.04.032

Rosenblum, M., Pikovsky, A., Kurths, J., Schäfer, C., and Tass, P. A. (2001). Phase synchronization: from theory to data analysis. Handb. Biol. Phys. 4, 279-321. doi: 10.1016/S1383-8121(01)80012-9

Schack, B., Vath, N., Petsche, H., Geissler, H. G., and Möller, E. (2002). Phasecoupling of theta-gamma EEG rhythms during short-term memory processing. Int. J. Psychophysiol. 44, 143-163. doi: 10.1016/S0167-8760(01)00199-4

Scherg, M., Ille, N., Bornfleth, H., and Berg, P. (2002). Advanced tools for digital EEG review: virtual source montages, whole-head mapping, correlation, and phase analysis. J. Clin. Neurophysiol. 19, 91-112. doi: 10.1097/00004691200203000-00001

Schlee, W., Mueller, N., Hartmann, T., Keil, J., Lorenz, I., and Weisz, N. (2009). Mapping cortical hubs in tinnitus. BMC Biol. 7:80. doi: 10.1186/1741-7007-7-80

Schlee, W., Weisz, N., Bertrand, O., Hartmann, T., and Elbert, T. (2008). Using auditory steady state responses to outline the functional connectivity in the tinnitus brain. PLoS ONE 3:e3720. doi: 10.1371/journal.pone.0003720

Seki, S., and Eggermont, J. J. (2003). Changes in spontaneous firing rate and neural synchrony in cat primary auditory cortex after localized tone-induced hearing loss. Hear. Res. 180, 28-38. doi: 10.1016/S0378-5955(03)00074-1

Shils, J. L., Litt, M., Skolnick, B. E., and Stecker, M. M. (1996). Bispectral analysis of visual interactions in humans. Electroencephalogr. Clin. Neurophysiol. 98, 113-125. doi: 10.1016/0013-4694(95)00230-8

Siapas, A. G., Lubenov, E. V., and Wilson, M. A. (2005). Prefrontal phase locking to hippocampal theta oscillations. Neuron 46, 141-151. doi: 10.1016/j.neuron.2005.02.028

Siegel, M., Donner, T. H., and Engel, A. K. (2012). Spectral fingerprints of large-scale neuronal interactions. Nat. Rev. Neurosci. 13, 121-134. doi: $10.1038 / \mathrm{nrn} 3137$ 
Silchenko, A. N., Adamchic, I., Hauptmann, C., and Tass, P. A. (2013). Impact of acoustic coordinated reset neuromodulation on effective connectivity in a neural network of phantom sound. Neuroimage 77C, 133-147. doi: 10.1016/j.neuroimage.2013.03.013

Snow, J. B. (2004). Tinnitus: Theory and Management. Hamilton; London: BC Decker.

Tass, P. A. (1995). Phase and frequency shifts of two nonlinearly coupled oscillators. Z. Phys. B 99, 111-121. doi: 10.1007/s002570050017

Tass, P. A. (1997). Phase and frequency shifts in a population of phase oscillators. Phys. Rev. E 56, 2043-2060. doi: 10.1103/PhysRevE.56.2043

Tass, P. A. (2003). A model of desynchronizing deep brain stimulation with a demand-controlled coordinated reset of neural subpopulations. Biol. Cybern. 89, 81-88. doi: 10.1007/s00422-003-0425-7

Tass, P. A., Adamchic, I., Freund, H. J., von Stackelberg, T., and Hauptmann, C. (2012). Counteracting tinnitus by acoustic coordinated reset neuromodulation. Restor. Neurol. Neurosci. 30, 137-159. doi: 10.3233/RNN-2012-110218

Tass, P. A., and Popovych, O. V. (2012). Unlearning tinnitus-related cerebral synchrony with acoustic coordinated reset stimulation-theoretical concept and modelling. Biol. Cybern. 106, 27-36. doi: 10.1007/s00422-012-0479-5

Tass, P. A., Rosenblum, M. G., Weule, J., Kurths, J., Pikovsky, A., Volkmann, J., et al. (1998). Detection of $\mathrm{n}$ : $\mathrm{m}$ phase locking from noisy data: application to magnetoencephalography. Phys. Rev. Lett. 81, 3291-3294. doi: 10.1103/PhysRevLett.81.3291

Tierney, P. L., Dégenètais, E., Thierry, A. M., Glowinski,. J., and Gioanni, Y. (2004) Influence of the hippocampus on interneurons of the rat prefrontal cortex. Eur J. Neurosci. 20, 514-524. doi: 10.1111/j.1460-9568.2004.03501.x

Tort, A. B. L., Komorowski, R., Eichenbaum, H., and Kopell, N. (2010). Measuring phase-amplitude coupling between neuronal oscillations of different frequencies. J. Neurophysiol. 104, 1195-1210. doi: 10.1152/jn.00106.2010

Tort, A. B. L., Kramer, M. A., Thorn, C., Gibson, D. J., Kubota, Y., Graybiel, A. M. et al. (2008). Dynamic cross-frequency couplings of local field potential oscillations in rat striatum and hippocampus during performance of a T-maze task. Proc. Natl. Acad. Sci. U.S.A. 105, 20517-20522. doi: 10.1073/pnas.0810524105

van der Loo, E., Gais, S., Congedo, M., Vanneste, S., Plazier, M., Menovsky, T., et al. (2009). Tinnitus intensity dependent gamma oscillations of the contralateral auditory cortex. PLoS ONE 4:e7396. doi: 10.1371/journal.pone.0007396

Vanneste, S., Plazier, M., van der Loo, E., Van de Heyning, P., and De Ridder, D. (2010). The differences in brain activity between narrow band noise and pure tone tinnitus. PLoS ONE 5:e13618. doi: 10.1371/journal.pone.0013618

Vanneste, S., van de Heyning, P., and De Ridder, D. (2011). The neural network of phantom sound changes over time: a comparison between recent-onset and chronic tinnitus patients. Eur. J. Neurosci. 34, 718-731. doi: 10.1111/j.14609568.2011.07793.x

von Stein, A., and Sarnthein, J. (2000). Different frequencies for different scales of cortical integration: from local gamma to long range alpha/theta synchronization. Int. J. Psychophysiol. 38, 301-313. doi: 10.1016/S0167-8760(00)00172-0

Voytek, B., Canolty, R. T., Shestyuk, A., Crone, N. E., Parvizi, J., and Knight, R. T. (2010). Shifts in gamma phase-amplitude coupling frequency from theta to alpha over posterior cortex during visual tasks. Front. Hum. Neurosci. 4:191. doi: 10.3389/fnhum.2010.00191

Weisz, N., Hartmann, T., Dohrmann, K., Schlee, W., and Norena, A. (2006). High-frequency tinnitus without hearing loss does not mean absence of deafferentation. Hear. Res. 222, 108-114. doi: 10.1016/j.heares.2006. 09.003

Weisz, N., Moratti, S., Meinzer, M., Dohrmann, K., and Elbert, T. (2005). Tinnitus perception and distress is related to abnormal spontaneous brain activity as measured by magnetoencephalography. PLoS Med. 2:e153. doi: 10.1371/journal.pmed.0020153

Weisz, N., Muller, S., Schlee, W., Dohrmann, K., Hartmann, T., and Elbert, T. (2007). The neural code of auditory phantom perception. J. Neurosci. 27, 1479-1484. doi: 10.1523/JNEUROSCI.3711-06.2007

Zatorre, R. J., Evans, A. C., and Meyer,. E. (1994). Neural mechanisms underlying melodic perception and memory for pitch. J. Neurosci. 14, 1908-1919.

Conflict of Interest Statement: Ilya Adamchic reports no commercial or financial relationships that could be construed as a potential conflict of interest. Berthold Langguth received honoraria and speakers' fee from ANM, Astra Zeneca, Autifony, Lundbeck, Merz, Magventure, Novartis, Pfizer and Servier, research funding from the Tinnitus Research Initiative, the German Research Foundation, the German Bundesministerium für Bildung und Forschung, the American Tinnitus Association, Astra Zeneca and Cerbomed, funding for equipment from Magventure and travel and accommodation payments from Medtronic, Lilly, Servier and Pfizer. Christian Hauptmann: employment Jülich Research Center \& former employment ANM GmbH. Christian Hauptmann has received research funding from the European Community, the Federal Ministry of Education and Research (Germany), the Deutsche Forschungsgemeinschaft, the Helmholtz Association. Peter A. Tass: employment Jülich Research Center; worked with ANM GmbH (Cologne, Germany) to develop devices for CR neuromodulation in patients, shareholder of ANM GmbH. Peter A. Tass has received research funding from the European Community, the Federal Ministry of Education and Research (Germany), the Deutsche Forschungsgemeinschaft, the Helmholtz Association, Biomedical Primate, the Michael J. Fox Foundation.

Received: 28 April 2014; accepted: 19 August 2014; published online: 25 September 2014

Citation: Adamchic I, Langguth B, Hauptmann C and Tass PA (2014) Abnormal crossfrequency coupling in the tinnitus network. Front. Neurosci. 8:284. doi: 10.3389/fnins. 2014.00284

This article was submitted to Auditory Cognitive Neuroscience, a section of the journal Frontiers in Neuroscience.

Copyright (c) 2014 Adamchic, Langguth, Hauptmann and Tass. This is an openaccess article distributed under the terms of the Creative Commons Attribution License (CC BY). The use, distribution or reproduction in other forums is permitted, provided the original author(s) or licensor are credited and that the original publication in this journal is cited, in accordance with accepted academic practice. No use, distribution or reproduction is permitted which does not comply with these terms. 